\title{
Rechtsklarheit tut not für Mobilität und Transport in Europa! Der Fall der Verordnung 169/2009
}

\author{
Erik Staebe
}

\section{Einleitung: Kooperation im internationalen Eisenbahnverkehr}

\begin{abstract}
Mobilität und Transport in Europa unterliegen vielfältigen rechtlichen Anforderungen. Dies gilt nicht nur für sämtliche Verkehrsträger, sondern es bezieht sich auch auf zahlreiche Materien des nationalen und des europäischen Rechts. Dieser Befund ist weder überraschend noch beklagenswert. Die historische Entwicklung des Verkehrswesens, seine Sicherheitsrelevanz sowie die volkswirtschaftliche und politische Bedeutung funktionierender Verkehrsinfrastruktur haben nicht zuletzt deshalb in Rechtssetzung, Rechtsanwendung und Rechtsprechung ihre Spuren hinterlassen, weil allgemeine Regelungen in vielen Fällen nicht ohne Weiteres auf den Verkehrssektor übertragen werden konnten. Die Besonderheiten des Verkehrs und die weiter gehenden Besonderheiten der einzelnen Verkehrsträger haben immer wieder zu sektorspezifischen Regelungen Anlass gegeben, die zu allgemeinen Vorschriften in ein Spannungsverhältnis treten.
\end{abstract}

Article note: Eric Staebe ist Leiter Kartellrechtliche Verfahren und Regulierungsrecht der Deutschen Bahn AG. Der Verfasser bringt in diesem Beitrag allein seine persönliche Auffassung zum Ausdruck.

E. Staebe $(\bowtie)$

Leiter Kartellrechtliche Verfahren und Regulierungsrecht, Deutsche Bahn AG,

Berlin, Deutschland

E-Mail: Erik.Staebe@deutschebahn.com

S. Laimer, C. Perathoner (Hrsg.), Mobilitäts- und Transportrecht in Europa,

Bibliothek des Wirtschaftsrechts 2,

https://doi.org/10.1007/978-3-662-63635-0_8 
Eine sektorspezifische Besonderheit des Eisenbahnsektors besteht beispielsweise darin, dass grenzüberschreitende Verkehre in Europa bis heute in aller Regel nicht im Wettbewerb, sondern im Rahmen von Kooperationen durchgeführt werden. ${ }^{1}$ Dies gilt sowohl für den Personenverkehr, der traditionell im Mittelpunkt der verkehrspolitischen Diskussion steht. Auch im grenzüberschreitenden Schienengüterverkehr sind Kooperationen durchaus üblich. Dabei sind sie weniger Ausdruck einer wettbewerbsfeindlichen Einstellung der beteiligten Unternehmen. Sie ergeben sich vielmehr aus betrieblich-praktischen Gründen, betriebswirtschaftlichen Erwägungen oder verkehrlichen Notwendigkeiten. So ermöglichen Kooperationen im Personenverkehr es den beteiligten Unternehmen, ihren Endkunden dichtere Taktfrequenzen, einheitliche Tarife und bessere Anschlussverbindungen in den beteiligten Ländern anzubieten als dies im Wettbewerb möglich wäre. Im Bereich des Schienengüterverkehrs, in dem zusätzlich zwischen dem Verkehr mit Ganzzügen und dem Einzelwagen- (ladungs-)verkehr zu unterscheiden ist, ergibt sich ein ähnliches Bild. Von Ganzzugverkehr spricht man, wenn ein Eisenbahnverkehrsunternehmen (EVU) einen kompletten Güterzug von einem Abfahrtspunkt zu einem Zielpunkt, etwa von einem Gleisanschluss zu einem anderen Gleisanschluss transportiert. Die Zusammenstellung des Zuges bleibt dabei von Anfang bis Ende unverändert. Die Leistung des EVU besteht allein in der Traktion des Zuges. Auch im Einzelwagenverkehr werden komplette Züge transportiert, allerdings typischerweise nur zwischen verschiedenen Rangierbahnhöfen, in denen die Züge aus Wagen mit unterschiedlichen Abfahrts- und Zielpunkten zusammengestellt werden. Über den Transport der Züge von einem Rangierbahnhof zum anderen hinaus müssen die Wagen von verschiedenen Abfahrtpunkten, etwa Gleisanschlüssen, Terminals oder anderen Güterverkehrsstellen, zu den Rangierbahnhöfen gebracht bzw. von dort wieder auf verschiedene Zielpunkte verteilt werden. Die vom EVU erbrachte Leistung ist im Vergleich zum Transport von Ganzzügen ungleich komplexer. Sie umfasst sowohl die Traktion des Zuges von einem Rangierbahnhof zum anderen als auch die Abholung und Zustellung der einzelnen Wagen sowie zusätzliche logistische Dienstleistungen, die dieses Produktionssystem mit sich bringt. Diese Leistungen verlangen von den EVU zusätzliche Ressourcen, die gerade im grenzüberschreitenden Verkehr in der Regel nur im Rahmen von Kooperationen verfügbar sind. Die Kunden der EVU, die mit den zusätzlichen logistischen Dienstleistungen nicht unmittelbar befasst sind, erwarten demgegenüber von den beauftragten EVU eine einheitliche Transportleistung zu einheitlichen Bedingungen und einem einzigen Preis.

Partner der hier beschriebenen Kooperationen sind regelmäßig auch und gerade diejenigen Unternehmen, die als ehemalige Staatsbahnen und Marktführer in ihren Heimatmärkten als potenzielle oder tatsächliche Wettbewerber in Betracht kommen. Kooperationen stellen im Prinzip horizontale Vereinbarungen zwischen Wettbewerbern dar, die nach dem in Art. 101 AEUV normierten Kartellverbot des europäischen Primärrechts und den ergänzenden Rechtsakten des Sekundärrechts zu

\footnotetext{
${ }^{1}$ Kritisch hierzu insbesondere Monopolkommission, s. zuletzt 7. Sektorgutachten Bahn „Mehr Qualität und Wettbewerb auf der Schiene“, 2019, S. 16 f.
} 
beurteilen sind. ${ }^{2}$ Für die Beantwortung der Frage, ob eine Kooperation von Unternehmen im grenzüberschreitenden Eisenbahnverkehr gegen die Verbotsvorschriften verstößt, ist in jedem Einzelfall zunächst zu prüfen, ob die beteiligten Unternehmen überhaupt in einem tatsächlichen oder potenziellen Wettbewerbsverhältnis stehen. ${ }^{3}$ Für ihre Beantwortung kommt es auf die ökonomischen Verhältnisse im Einzelfall an. Besteht nicht wenigstens ein potenzielles Wettbewerbsverhältnis, dürfen die Kooperationspartner ggf. eine kartellrechtlich zulässige „Arbeitsgemeinschaft" bilden. ${ }^{4}$ Andernfalls würde der Verbotstatbestand der Art. 101 AEUV eingreifen. Dann ist zu prüfen, ob die Anwendung des Kartellverbots nicht (auch) aus Rechtsgründen ausscheidet, etwa weil das europäische Kartellrecht für die Kooperation im grenzüberschreitenden Eisenbahnverkehr spezielle Ausnahmeregelungen enthält. Einen solchen Weg könnte die weitgehend unbekannte und in der Praxis wenig bedeutsame Verordnung (EG) 169/2009, ,über die Anwendung von Wettbewerbsregeln auf dem Gebiet des Eisenbahn-, Straßen- und Binnenschiffsverkehrs" ${ }^{\text {5 }}$ eröffnen. Ihre Entstehungsgeschichte (hierzu u. 2.) und eine Analyse der dortigen Regelungen (hierzu u. 3. und 4.) führt allerdings zu einem ernüchternden Befund: Es besteht erhebliche Unsicherheit über den materiellen Gehalt und die Reichweite der dort enthaltenen Ausnahmevorschriften (hierzu u. 5.).

Der europäische Schienenverkehr gilt heute als fester Bestandteil der Klimapolitik. Die im Pariser Klimaabkommen verankerte Reduktion der Treibhausgase im Verkehr dürfte nur gelingen, wenn die Mobilität klimafreundlicher wird. Als Antwort auf diese Herausforderungen gilt nicht zuletzt ein besseres europäisches Eisenbahnverkehrsangebot. Dass die Umsetzung notwendiger Maßnahmen bisher nur schleppend verläuft, könnte auch an unklaren und unzureichenden rechtlichen Regelungen liegen. Auch insofern besteht also Handlungsbedarf für Mobilität und Transport in Europa.

\section{Hintergrund der Verordnung (EG) Nr. 169/2009}

Die Entstehungsgeschichte der Verordnung (EG) Nr. 169/2009 ist eng mit der Entstehung des europäischen Kartellverfahrensrechts verbunden. Nachdem derartige Vorschriften im ursprünglichen EWG-Vertrag fehlten, kam es erstmals im Jahre

\footnotetext{
${ }^{2}$ Zur Anwendung der Wettbewerbsregeln im Verkehrssektor EuGH, Urteil vom 30.4.1986, verb. Rs. 209-213/84, ECLI:EU:C:1986:188 - Nouvelles Frontieres, Tz. 35-42.

${ }^{3}$ St. Verwaltungspraxis und Rspr., vgl. nur EuG, Urteil vom 29.6.2012, Rs. T-360/09 - E.ON Ruhrgas AG \& E.ON AG/Kommission, Tz. 86 m.w.N.; Urteile vom 15.9.1998, Rs. T-374/94, T-375/94, T-384/94 und T-388/94 - European Night Services/Kommission, Tz. 137; Kommission, Leitlinien zur Anwendbarkeit von Artikel 101 des Vertrages über die Arbeitsweise der Europäischen Union auf Vereinbarungen über horizontale Zusammenarbeit, ABl. 2011 C 11, S. 1, Rn. 2.

${ }^{4}$ Vgl. hierzu ausf. Emmerich, Kartellrecht, 13. Aufl. 2014, § 5 Rn. 9 und § 21 Rn. 36 f.

${ }^{5}$ Verordnung (EG) Nr. 169/2009 des Rates vom 26.2.2009 über die Anwendung von Wettbewerbsregeln auf dem Gebiet des Eisenbahn-, Straßen- und Binnenschiffsverkehrs (kodifizierte Fassung), AB1. 2009 L 61, S. 1.
} 
1962 zum Erlass einer Kartellverfahrensverordnung, ${ }^{6}$ von deren Anwendung der Verkehrssektor allerdings gleich wieder ausgenommen wurde. ${ }^{7}$ Durchführungsvorschriften für das Kartellverbot und das Verbot des Missbrauchs einer marktbeherrschenden Stellungen im Verkehrssektor wurden erst mit der Verordnung (EWG) Nr. 1017/68 erlassen. ${ }^{8}$ Bei dieser Verordnung handelte es sich um eine Maßnahme der europäischen Wettbewerbspolitik und der gemeinsamen Verkehrspolitik. ${ }^{9}$ Sie beruhte sowohl auf Verordnungsermächtigungen des Wettbewerbsrechts im damaligen Art. 87 EWGV als auch der Verkehrspolitik im früheren Art. 75 EWGV. Sie etablierte ein gesondertes Wettbewerbsrecht für die Verkehrsmärkte und nahm insofern von Anfang an eine Sonderstellung unter den Rechtsakten des sekundären Wettbewerbsrechts ein. Nachdem der Europäische Gerichtshof im Jahre 1986 festgestellt hatte, dass die europäischen Wettbewerbsregeln auch auf den Verkehrssektor angewendet werden müssen, ${ }^{10}$ war dem gesonderten ,Verkehrskartellrecht“ der Verordnung (EWG) Nr. 1017/68 zunächst die Grundlage entzogen. Erst mit dem Erlass einer neuen Kartellverfahrensverordnung in Gestalt der VO 1/2003 ${ }^{11}$ wurden zahlreiche Regelungen der Verordnung (EWG) Nr. 1017/68 formell aufgehoben. ${ }^{12}$ Übrig blieben allerdings Ausnahmen vom Kartellverbot und die Erwägungsgründe, do dass schon bald nach Erlass der VO 1/2003 Überlegungen zur Bereinigung der verbliebenen Vorschriften der Verordnung (EWG) Nr. 1017/68 angestellt wurden. Nach dem entsprechenden Vorschlag der Kommission aus dem Jahre 2006 sollten vor allem die Vorschriften neu nummeriert und überflüssige Erwägungsgründe gestrichen werden, um die Verordnung letztlich übersichtlicher und transparenter zu gestalten. ${ }^{13}$ Im Ergebnis führte dies zum Erlass der Verordnung (EG) Nr. 169/2009 des Rates vom 26. Februar 2009 über die Anwendung von Wettbewerbsregeln auf dem Gebiet des Eisenbahn-, Straßen- und Binnenschiffsverkehrs. ${ }^{14}$ Im Vergleich zu

\footnotetext{
${ }^{6}$ Verordnung (EWG) Nr. 17/1962 - Durchführungsverordnung zu Art. 85 und 86 des Vertrages vom 6.2.1962, AB1. 1962, S. 204.

${ }^{7}$ Verordnung (EWG) Nr. 141/1962 des Rates über die Nichtanwendung der Verordnung Nr. 17 des Rates auf den Verkehr vom 26.11.1962, AB1. 1962, S. 2751.

${ }^{8}$ Verordnung (EWG) Nr. 1017/1968 des Rates über die Anwendung von Wettbewerbsregeln auf dem Gebiet des Eisenbahn-, Straßen- und Binnenschiffsverkehrs vom 19.7.1968, ABl. 1968 L 175, S. 1.

${ }^{9}$ Vgl. hierzu den dritten Erwägungsgrund der Verordnung (EWG) Nr. 1017/1968.

${ }^{10} \mathrm{EuGH}$, Urteil vom 30.4.1986, verb. Rs. 209-213/84, ECLI:EU:C:1986:188 - Nouvelles Frontieres, Tz. 35-42.

${ }^{11}$ Verordnung (EG) Nr. 1/2003 des Rates vom 16.12.2002 zur Durchführung der in den Artikel 81 und 82 des Vertrags niedergelegten Wettbewerbsregeln, AB1. 2003 L 1, S. 1.

${ }^{12}$ Verordnung (EG) Nr. 169/2009 des Rates vom 26.2.2009 über die Anwendung von Wettbewerbsregeln auf dem Gebiet des Eisenbahn-, Straßen- und Binnenschiffsverkehrs (kodifizierte Fassung), AB1. 2009 L 61, S. 1.

${ }^{13} \mathrm{Vgl}$. Kommission, Vorschlag für eine Verordnung des Europäischen Parlaments und des Rates über die Anwendung von Wettbewerbsregeln auf dem Gebiet des Eisenbahn-, Straßen- und Binnenschiffsverkehrs (kodifizierte Fassung) vom 27.11.2006, KOM(2006) 722 endg., S. 2 f.

${ }^{14}$ Der Erlass der Verordnung erfolgte im sog. beschleunigten Verfahren, das nach einer interinstitutionellen Vereinbarung der am Rechtssetzungsverfahren beteiligten Organe für redaktionelle Bereinigungen der Rechtslage vorgesehen ist. (Wesentliche) inhaltliche Änderungen sind in einem
} 
den verbliebenen Regelungen der Vorgängerregelung wurden keine wesentlichen inhaltlichen Änderungen der früheren Verordnung - soweit diese nach Erlass der VO 1/2003 noch in Kraft war - vorgenommen.

\section{Regelungsgehalt der Verordnung (EG) Nr. 169/2009}

Die Verordnung beginnt mit den Erwägungsgründen, in denen die historischen und systematischen Hintergründe erläutert werden. U. a. wird klargestellt, dass Wettbewerbsregeln für den Eisenbahn-, Straßen- und Binnenschiffsverkehr sowohl Teil der Verkehrs- als auch der allgemeinen Wirtschaftspolitik (bzw. Wettbewerbspolitik) darstellen - mit der Folge, dass die Wettbewerbsregeln den Besonderheiten des Verkehrs Rechnung tragen sollten. ${ }^{15}$ Zudem wird betont, dass die Wettbewerbsregeln für den Verkehrssektor von den allgemeinen Wettbewerbsregeln abweichen. ${ }^{16}$ Hier wird auf die Möglichkeit verwiesen, bestimmten Formen der technischen Zusammenarbeit vom Kartellverbot auszunehmen ${ }^{17}$ die inhaltliche Beurteilung müssten die Unternehmen - im System der „Legalausnahme“ mit dem Zwang zur „Selbstveranlagung" nach der VO 1/2003 - letztlich selbst vornehmen, ohne die fraglichen Vereinbarungen bekannt geben zu müssen. ${ }^{18}$

In Erwägung dieser Gesichtspunkte bestimmt Art. 1 der Verordnung zunächst als Anwendungsbereich der Verordnung den Eisenbahn-, Straßen- und Binnenschiffsverkehr. Art. 2 Abs. 1 der Verordnung enthält ,gesetzliche Ausnahme für die technischen Vereinbarungen". So findet das Kartellverbot nach dem Eingangssatz des Artikels auf Vereinbarungen, Beschlüsse und aufeinander abgestimmte Verhaltensweisen keine Anwendung, wenn diese ,ausschließlich die Anwendung technischer Verbesserungen oder die technische Zusammenarbeit bezwecken und bewirken“ Wie die Formulierung ,und zwar durch“ anzeigt, folgen danach Beispiele für Maßnahmen, die ausschließlich der technischen Verbesserung oder der technischen Zusammenarbeit dienen. Genannt werden die einheitliche Anwendung von Normen und Typen für Material (lit. a), die gemeinsame Nutzung von Personal, Material, Fahrzeugen oder Einrichtungen (lit. b), „die Regelung und Durchführung von Anschlussbeförderungen, ergänzenden Beförderungen, Ersatzbeförderungen oder kombinierten Beförderungen sowie die Aufstellung und Anwendung von Gesamtpreisen und Gesamtbedingungen einschließlich Wettbewerbspreisen auf diese Beförderungen“ (lit. c); eine zweckmäßige Verkehrslenkung (lit. d), die Abstimmung von Fahrplänen (lit. e), eine Zusammenfassung von Einzelladungen (lit. f) und die Aufstellung einheitlicher Regeln für die Struktur der Beförderungstarife (lit. g).

\footnotetext{
solchen Verfahren ausgeschlossen; vgl. Vereinbarung über ein beschleunigtes Arbeitsverfahren, AB1. 1996 C 102, S. 2.

${ }^{15}$ Vgl. hierzu den zweiten und dritten Erwägungsgrund der Verordnung (EG) Nr. 169/2009.

${ }^{16}$ Vgl. hierzu den vierten Erwägungsgrund der Verordnung (EG) Nr. 169/2009.

${ }^{17}$ Vgl. hierzu den siebten Erwägungsgrund der Verordnung (EG) Nr. 169/2009.

${ }^{18}$ Vgl. hierzu den neunten und zehnten Erwägungsgrund der Verordnung (EG) Nr. 169/2009.
} 
Nach Art. 2 Abs. 2 kann die Kommission dem Rat ggf. Vorschläge zur Ausweitung oder Kürzung dieser Aufzählung unterbreiten. Während sich die Ausnahme des Art. 2 noch auf sämtliche Verkehrssektoren bezieht, gewährt Art. 3 der Verordnung weiter gehende Ausnahmen für Gemeinschaften kleiner und mittlerer Unternehmen im Bereich des Straßen- und Binnenschiffsverkehrs. Art. 4 und Art. 5 der Verordnung enthalten Schlussbestimmungen zur formellen Aufhebung der Verordnung (EWG) Nr. 1017/68, zum Inkrafttreten der Nachfolgeregelung und zum Umgang mit „Altkartellen“.

\section{Internationale Kooperation nach Art. 2 Abs. 1 der Verordnung (EG) Nr. 169/2009}

Die für die grenzüberschreitende Kooperation im Schienenverkehr entscheidende Vorschrift der Verordnung ist Art. 2 Abs. 1. Sie stimmt vom Wortlaut her im Wesentlichen mit Art. 3 Abs. 1 der Verordnung (EWG) Nr. 1017/68 überein. Die Vorschrift ist als Legalausnahme formuliert, nicht als eine konstitutiv wirkende Freistellung. Nach der Umstellung des europäischen Kartellverfahrensrechts auf das System der Legalausnahme dürften sich hieraus allerdings kaum noch Unterschiede ergeben, weil auch die Freistellung nach Art. 101 Abs. 3 AEUV unmittelbar anwendbar ist und faktisch eine Legalausnahme mit unbestimmten Tatbestandsvoraussetzungen darstellt. Von den Art. 101 Abs. 3 AEUV konkretisierenden Freistellungsverordnungen unterscheidet sich die Legalausnahme nach Art. 2 Abs. 1 auch dadurch, dass sie als zeitlich unbefristete Regelung vom Rat erlassen wurde. Freistellungsverordnung ergehen demgegenüber zeitlich befristet durch die Kommission, die sich insoweit auf eine vom Rat erlassene Verordnungsermächtigung stützt. Insoweit folgt die Verordnung (EG) Nr. 169/2009 ihrer Vorgängerregelung. Hier zeigt sich zudem der enge Zusammenhang mit der gemeinsamen Verkehrspolitik, die sich nicht auf den Freistellungstatbestand vom Kartellverbot, sondern auf eine eigene vertragliche Ermächtigungsgrundlage stützte.

Das zentrale Regelungskonzept des Art. 2 Abs. 1 liegt in der Ausnahme für „technische“ Vereinbarungen bzw. „technische“ Verbesserungen und die ,technische“ Zusammenarbeit. Die Bezugnahme auf „technische“ Vorgänge findet sich sowohl in der Überschrift zu Art. 2 als auch im Eingangssatz des Artikels. Dies deutet darauf hin, dass die im Folgenden genannten Beispiele (lit. a bis lit. g) alle einen „technischen“ Charakter aufweisen müssen. In der deutschen Fassung der Verordnung wird das Gewicht des Begriffs noch verstärkt, indem die betreffende Vereinbarung ,ausschließlich“ hierauf gerichtet sein muss. Ein Vergleich dieser Regelung mit anderen Sprachfassungen und mit der Vorgängerregelung in der Verordnung (EWG) Nr. 1017/68 liefert hingegen ein weniger eindeutiges Bild. Weder die aktuelle französische noch die englische Sprachfassung der aktuellen Verordnung enthalten eine Entsprechung zum deutschen Begriff ,ausschließlich“. Er fand sich bereits in der deutschen Vorgängerregelung. Auch die französische Vorgängerrege- 
lung verlangte die „seulement“ technische Natur der fraglichen Vereinbarungen. In der englischen Fassung der Vorgängerregelung fehlte es hingegen an diesem Erfordernis. ${ }^{19}$ Festzuhalten ist, dass bei Bereinigung und Neuerlass der alten Verordnung im Jahre 2009 das Ausschließlichkeitserfordernis zumindest in der französischen Sprachfassung weggefallen ist. Demnach sehen einzelne der heute gültigen Sprachfassungen eine Ausnahme nur für solche Vereinbarungen vor, die einen ausschließlich technischen Charakter haben, während andere Sprachfassungen die Ausnahme auch bei Vereinbarungen zulassen, die andere Zwecke verfolgen. Zur Vermeidung von Auslegungsschwierigkeiten kommt es für die Auslegung mehrsprachiger Verträge nach überwiegender Auffassung auf den Wortlaut nicht entscheidend an. ${ }^{20}$ Verschiedene Sprachfassung müssen ggf. im Wege einer systematisch-teleologischen Auslegung der betreffenden Vorschriften in Einklang gebracht werden. In systematischer Hinsicht fällt dabei vor allem auf, dass die Regelung unter lit. c u. a. „die Aufstellung und Anwendung von Gesamtpreisen und Gesamtbedingungen einschließlich Wettbewerbspreisen" (Hervorhebung nur hier) umfasst. Eine rein technische Zusammenarbeit bei der Aufstellung von Wettbewerbspreisen erscheint praktisch nicht möglich: Preise werden entweder in Zusammenarbeit aufgestellt. Dann handelt es sich nicht mehr um Wettbewerbspreise. Sonst müssten sie unabhängig voneinander aufgestellt werden. Dann findet aber keine Zusammenarbeit mehr statt, und zwar auch nicht in „technischer" Hinsicht. Das systematische Nebeneinander des Begriffs der ausschließlich „technischen“ Zusammenarbeit und der gemeinsamen Aufstellung von „Wettbewerbspreisen“ führt folglich zu einem Widerspruch in der Regelung, der sich im Hinblick auf die Unklarheit der divergierenden Sprachfassungen vorrangig dadurch auflösen lässt, dass auf das Kriterium der ,ausschließlich" technischen Zusammenarbeit verzichtet werden muss.

Eine andere Interpretation könnte sich demgegenüber aus einer älteren Entscheidungen des Gerichts Erster Instanz und des Europäischen Gerichtshofs zu Art. 3 der Verordnung (EWG) Nr. 1017/68 ergeben. Hier war ein Preisfestsetzungsmechanismus mehrerer staatlicher Güterbahnen für den Hinterlandverkehr verschiedener Seehäfen an der Nordsee zu beurteilen. Die Kommission hatte einen Verstoß gegen das Kartellverbot festgestellt, ${ }^{21}$ den das Gericht bestätigte. Die Anwendung der Legalausnahme des Art. 3 Abs. 1 lit. c) der Verordnung (EWG) Nr. 1017/68 wurde sowohl unter Hinweis auf die Privilegierung von Vereinbarungen rein technischer Natur nach dem Wortlaut der Vorschrift als auch im Hinblick auf den Sinn und Zweck der Normen abgelehnt: Die Wettbewerbsregeln der Vertrages zielten darauf ab, dass jeder Unternehmer seine Preispolitik selbst bestimme. Vor diesem Hintergrund müssten Begriffe wie „Wettbewerbspreise“"zurückhaltend ausgelegt werden. ${ }^{22}$

\footnotetext{
${ }^{19} \mathrm{Vgl}$. Basedow, in Immenga/Mestmäcker, Kommentar zum europäischen Wettbewerbsrecht, 4. Aufl., Verkehr, Rn. 21.

${ }^{20} \mathrm{EuGH}$, Urteil vom 22.6.2016, Rs. C-207/15 P, ECLI:EU:C:2016:465 Rn. 43 m.w.N.

${ }^{21}$ Kommission, Entscheidung vom 29.3.1994 (IV/33.941 HOV-SVZ/MCN), ABl. 1994 L 104/34.

${ }^{22} \mathrm{EuG}$, Urteil vom 21.10.1997, Rs. T-229/94, ECLI:EU:T:1997:155, Rn. 37 f. - Deutsche Bahn ./. Kommission - Maritime Container Network; ebenso EuGH, Urteil vom 27.4.1999, Rs. C-436/97P, ECLI:EU:C:1999:205.
} 
Die Entscheidung hat im Schrifttum in der Folgezeit zu der Schlussfolgerung geführt, dass die Ausnahmetatbestände der Verordnung (EG) Nr. 169/2009 keine konstitutive Ausnahme vom Kartellverbot begründeten, sondern lediglich klarstellten, dass z. B. Kooperationen bereits in Anwendung der allgemeinen Regeln (z. B. nach dem „Arbeitsgemeinschaftsgedanken“) möglich seien. ${ }^{23}$

\section{Offene Fragen und Klarstellungsbedarf}

Offen bleibt, ob die Diskussion über die Bedeutung und Reichweite der Verordnung (EG) Nr. 169/2009 damit bereits abgeschlossen ist. Zunächst hat das Gericht Erster Instanz lediglich entschieden, dass der verfahrensgegenständliche Preisfestsetzungsmechanismus eine kartellrechtswidrige Absprache darstellte, auf die die damalige Ausnahmeregelung keine Anwendung fand. $\mathrm{Zu}$ der Frage, welche technischen Vereinbarungen gerade im Zusammenspiel mit der Festsetzung von Wettbewerbspreisen im Rahmen der Ausnahmeregelung demgegenüber zulässig sind, hat sich das Gericht nicht geäußert. Dies mag daran liegen, dass der Begriff der „Technik“ zwar oft verwendet wird, inhaltlich aber nicht klar konturiert ist. Infolgedessen ist in der Literatur der Versuch unternommen worden, Vereinbarungen als rein ,technische“ Vereinbarungen zu qualifizieren, die die Zusammenarbeit auf die verfahrensmäßige Durchführung von Nutzensteigerung, also auf Rationalisierungen beschränken und sie nicht auf den geschäftlichen Umgang mit dem Ergebnis der Rationalisierung erstrecken. ${ }^{24}$ Tatsächlich enthalten einzelne Ausnahmetatbestände des Katalogs in Art. 2 Abs. 1 lit. a bis lit. g den Gedanken der Rationalisierung. Gleichwohl ist zweifelhaft, ob es einer Ausfüllung des inhaltlich unklar konturierten Begriffs der „technischen“ Vereinbarungen bedarf. Sie ist vielmehr in Art. 2 Abs. 1 bereits enthalten. Die Vorschrift legt bereits fest, dass die in Art. 2 Abs. 1 lit. a bis lit. g genannten Vereinbarungen ,technische“ Vereinbarungen sind, auch wenn die Parteien darüber hinaus auch andere Ziele verfolgen. Letztlich ist der Begriff der „technischen“ Vereinbarung kein eigenständiges Tatbestandsmerkmal, das losgelöst vom Katalog der in Art. 2 Abs. 1 lit. a bis lit. g genannten Vereinbarungen zu prüfen wäre. ${ }^{25}$ Bei der Prüfung einer Vereinbarung anhand der Verordnung ist die Frage zu stellen, ob die Vereinbarung unter einen der sieben Katalogtatbestände fällt. In diesem Fall steht fest, dass durch sie eine technische Kooperation bezweckt bzw. bewirkt wird.

\footnotetext{
${ }^{23}$ So die wohl h.M., vgl. nur Wachinger/Herrmann/Kreis, in Loewenheim/Meesen/Riesenkampff/ Kersting/Meyer-Lindemann, Kartellrecht, 3. Aufl. 2016, Landverkehr und Binnenschifffahrt, Rn. 28 ff. und 51; Knauff, in Immenga/Mestmäcker, Wettbewerbsrecht, 5. Aufl. 2016, VO 169/2009, Rn. 53; a. A. aber Rother/v. Merveldt, in Busch/Röhling, Kölner Kommentar, Bd. 4, Syst. I, Rn. 16.

${ }^{24} \mathrm{Vgl}$. Burmeister, Wettbewerb der Eisenbahnen, S. 220; kritisch hierzu Rother/v. Merveldt, in Busch/Röhling, Kölner Kommentar, Bd. 4, Syst. I, Rn. 16.

${ }^{25}$ Ähnlich Rother/v. Merveldt, in Busch/Röhling, Kölner Kommentar, Bd. 4, Syst. I, Rn. 16.
} 
Als Kontrollüberlegung mag die Frage angestellt werden, ob die Vereinbarung zusätzlich im „technischen“ Sinne der Effizienzverbesserung oder Rationalisierung dient. Wo Vereinbarungen die Voraussetzung dafür sind, dass die beteiligten Unternehmen die knappe Eisenbahninfrastruktur nutzen können (vgl. lit. a, lit. d oder lit. e), dürfte jedenfalls keine Beschränkung des Wettbewerbs für Verkehrsleistungen vorliegen. Bei anderen Vereinbarungen ist es allerdings denkbar, dass ihr Gegenstand auch mögliche Parameter des Wettbewerbs betrifft, so etwa bei der Vergemeinschaftung von Personal gemäß lit. b, wenn die beteiligten Unternehmen auf diese Weise Unterschiede in den Lohnkosten nivellieren, die ihnen aufgrund ihrer jeweiligen Tarifverträge sonst entstünden. Die Aufnahme dieser Art von Vereinbarung in die Liste des Art. 2 ist in manchen Fällen also eine konstitutive Freistellung. Die in lit. a bis lit. g genannten Typen von Vereinbarungen stehen jedenfalls außerhalb des Verbots des Art. 101 Abs. 1 AEUV, ohne dass es einer zusätzlichen Prüfung weiterer Elemente bedürfte. Es ist keine Entscheidung der Kommission oder Rechtsprechung ersichtlich, in der bzgl. eines der Ausnahmetatbestände in den lit. a bis lit. g noch zusätzlich geprüft worden wäre, ob es sich tatsächlich um eine ,,ausschließlich technische" Vereinbarung handelt. Dagegen wird der technische Charakter der ausgenommenen Verhaltensweise zum Teil bei der Auslegung einzelner Ausnahmetatbestände betont und als Begründung für eine enge Auslegung der Ausnahmeregelung des Art. 2 VO (EG) Nr. 169/2009 herangezogen. ${ }^{26}$

Auch wenn die Kooperation von EVU im grenzüberschreitenden Personen- und Güterverkehr auf der Schiene im Prinzip unter die Ausnahmetatbestände des Art. 2 VO (EG) Nr. 169/2009 fällt, bleibt das Ergebnis zumindest in einem System der Selbstveranlagung unbefriedigend. Es verbleibt eine nicht unerhebliche Unsicherheit über die grundsätzliche Anwendbarkeit der Ausnahmetatbestände, die Anwendbarkeit der Tatbestände im Einzelnen und die Frage, welche (Teile von) Vereinbarungen ggf. überschießend sein könnten und deshalb nicht mehr von der Ausnahme erfasst werden. So betrifft die Ausnahme des Art. 2 Abs. 1 lit. c Vereinbarungen über die Anschlussbeförderung und hierauf bezogene Preisvereinbarungen. Preisvereinbarungen sind Absprachen über „die Aufstellung und Anwendung von Gesamtpreisen und Gesamtbedingungen einschließlich Wettbewerbspreisen auf diese Beförderungen“. Die Ausnahme dürfte also nicht eingreifen, soweit es keine zugrunde liegende Beförderung gibt oder sich die Preisabsprache jedenfalls nicht auf eine solche Beförderung bezieht. Völlig eindeutig ist dies freilich nicht. Gleiches gilt für die Vereinbarung von Gesamtpreisen ,einschließlich Wettbewerbspreisen". Sie ist ihrerseits nur ausgenommen, wenn bereits die Anschlussbeförderung unter die Ausnahme fällt. Gäbe es für eine Anschlussbeförderung keine Preisvereinbarung, so würden sich die Preise aus einer Addition der für die einzelnen nationalen Teilstrecken tariflich vorgesehenen Teilpreise ergeben. Der Kunde hätte es dann in der Hand, mit den einzelnen beteiligten EVU Rabattierungen zu vereinbaren. Indem Art. 2 Abs. 1 lit. c die Vereinbarung von „Gesamtpreisen“ für die

${ }^{26} \mathrm{Vgl}$. Hubert de Broca/Marta Mielecka Riga/Anatoly Subočs in Jonathan Faull/Ali Nikpay, Hrsg., The EU Law of Competition, 3. Aufl. Oxford 2014, Rn. 15.249, S. 1838 f. 
gesamte durchgehende Beförderung gestattet, wird den Kunden diese Möglichkeit getrennter Preisverhandlungen abgeschnitten.

\section{Zusammenfassung}

Zusammenfassend ist festzuhalten, dass Gründe dafür sprechen, die in der VO (EG) Nr. 169/2009 enthaltene Legalausnahme über rein „technische“ Gegenstände hinaus zu erstrecken. Der für den Verkehr im europäischen Eisenbahnraum notwendige klare und eindeutige Rechtsrahmen wird hier aber noch nicht erreicht. Von Maßnahmen im Rahmen der Rechtsanwendung ist keine Abhilfe zu erwarten. Mit den geschilderten Auslegungsschwierigkeiten kämpfen sowohl die Kommission als auch die betroffenen Unternehmen. Denkbar wäre, dass die Kommission die ihr in Art. 2 Abs. 2 der Verordnung (EG) Nr. 169/2009 zugewiesene Befugnis nutzt, dem Rat „Vorschläge zur Ausweitung oder Kürzung der Aufzählung in Absatz 1“ zu unterbreiten. Der Katalog des Art. 2 Abs. 1 lit. a bis lit. g könnte auf diese Weise zwar erweitert und mit Klarstellungen versehen werden. Die Auslegungsfragen, ob hier ,ausschließlich technische“ Vereinbarungen erfasst sind oder was sich hinter dem Begriff der ,technischen“ Vereinbarung verbirgt, dürfte sich so letztlich nicht lösen lassen. Abhilfe dürfte erst möglich sein, wenn normhierarchisch auf Ebene der Kartellverfahrensverordnung eine Ratsverordnung geschaffen wird, die die Reichweite der „Anwendung der Wettbewerbsregeln auf dem Gebiet des Eisenbahn-, Straßen- und Binnenschiffsverkehrs" regelt. Aus der Verordnung (EG) Nr. 169/2009 des Kartellverbots ließen sich immerhin der Titel und weitere Einzelregelungen übernehmen, deren praktische Wirksamkeit derzeit an den dargestellten (formellen) Einwänden scheitert. Wenn Europa es mit den Pariser Klimazielen ernst meint, sollte dem Eisenbahnverkehr ein klarer Rechtsrahmen nicht vorenthalten werden.

Open Access Dieses Kapitel wird unter der Creative Commons Namensnennung 4.0 International Lizenz (http://creativecommons.org/licenses/by/4.0/deed.de) veröffentlicht, welche die Nutzung, Vervielfältigung, Bearbeitung, Verbreitung und Wiedergabe in jeglichem Medium und Format erlaubt, sofern Sie den/die ursprünglichen Autor(en) und die Quelle ordnungsgemäß nennen, einen Link zur Creative Commons Lizenz beifügen und angeben, ob Änderungen vorgenommen wurden.

Die in diesem Kapitel enthaltenen Bilder und sonstiges Drittmaterial unterliegen ebenfalls der genannten Creative Commons Lizenz, sofern sich aus der Abbildungslegende nichts anderes ergibt. Sofern das betreffende Material nicht unter der genannten Creative Commons Lizenz steht und die betreffende Handlung nicht nach gesetzlichen Vorschriften erlaubt ist, ist für die oben aufgeführten Weiterverwendungen des Materials die Einwilligung des jeweiligen Rechteinhabers einzuholen. 